\title{
Novel Immune Features of the Systemic Inflammation Associated with Primary Hypercholesterolemia: Changes in Cytokine/Chemokine Profile, Increased Platelet and Leukocyte Activation
}

\author{
Aida Collado $1,3,+$, Patrice Marques $1,3,+\left(\mathbb{D}\right.$, Elena Domingo ${ }^{1,+}$, Eva Perello ${ }^{3,4}(\mathbb{D}$, \\ Herminia González-Navarro ${ }^{3,4}$, Sergio Martinez-Hervás ${ }^{2,3,4}$, José T. Real ${ }^{2,3,4}$, Laura Piqueras ${ }^{1,3}$, \\ Juan F. Ascaso ${ }^{2,3,4, *}$ and Maria-Jesus Sanz 1,3,4,* \\ 1 Department of Pharmacology, Faculty of Medicine and Odontology, University of Valencia, \\ Av. Blasco Ibáñez 15, 46010 Valencia, Spain; aida.collado@uv.es (A.C.); patricegmarques@gmail.com (P.M.); \\ eledo2@hotmail.com (E.D.); laura.piqueras@uv.es (L.P.) \\ 2 Department of Medicine, Faculty of Medicine and Odontology, University of Valencia, Av. Blasco Ibáñez 15, \\ 46010 Valencia, Spain; Sergio.Martinez@uv.es (S.M.-H.); Jose.T.Real@uv.es (J.T.R.) \\ 3 Institute of Health Research of the University Clinic Hospital of Valencia (INCLIVA), \\ Av. Menéndez Pelayo 4, 46010 Valencia, Spain; evapc89@hotmail.com (E.P.); \\ Herminia.Gonzalez@uv.es (H.G.-N.) \\ 4 Diabetes and Associated Metabolic Diseases Networking Biomedical Research Centre (CIBERDEM), \\ Institute of Health Carlos III (ISCIII), Av. Monforte de Lemos 3-5, 28029 Madrid, Spain \\ * Correspondence: ascaso@uv.es (J.F.A.); maria.j.sanz@uv.es (M.-J.S.); Tel.: +34-963-864-629 (M.-J.S.) \\ $\dagger$ These authors contributed equally to this work.
}

Received: 19 November 2018; Accepted: 19 December 2018; Published: 22 December 2018

\begin{abstract}
Primary hypercholesterolemia (PH) is associated with a low grade systemic inflammation that is likely the main driver of premature atherosclerosis. Accordingly, we characterized the immune cell behaviour in $\mathrm{PH}$ and its potential consequences. Whole blood from $22 \mathrm{PH}$ patients and 21 age-matched controls was analysed by flow cytometry to determine the percentage of leukocyte immunophenotypes, activation, and platelet-leukocyte aggregates. Plasma markers were determined by Enzyme-Linked ImmunoSorbent Assay (ELISA). The adhesion of platelet-leukocyte aggregates to tumor necrosis factor- $\alpha(\mathrm{TNF} \alpha)$-stimulated arterial endothelium was investigated using the dynamic model of the parallel-plate flow chamber. $\mathrm{PH}$ patients presented greater percentage of Mon 3 monocytes, Th2 and Th17 lymphocytes, activated platelets, and leukocytes than controls. The higher percentages of circulating platelet-neutrophil, monocyte and lymphocyte aggregates in patients caused increased platelet-leukocyte adhesion to dysfunctional arterial endothelium. Circulating CXCL8, CCL2, CX 3 CL1, and IL-6 levels positively correlated with key lipid features of $\mathrm{PH}$, whereas negative correlations were found for IL-4 and IL-10. We provide the first evidence that increased platelet and leukocyte activation leads to elevated platelet-leukocyte aggregates in PH and augmented arterial leukocyte adhesiveness, a key event in atherogenesis. Accordingly, modulation of immune system behavior might be a powerful target in the control of further cardiovascular disease in $\mathrm{PH}$.
\end{abstract}

Keywords: primary hypercholesterolemia; cytokines; chemokines; leukocyte activation; platelet activation; endothelial dysfunction; systemic inflammation 


\section{Introduction}

Cardiovascular disease (CVD), predominantly coronary heart disease (CHD) and stroke, remains the main cause of death in most European countries [1], and atherosclerosis is the most common pathologic process for myocardial and cerebral ischemic disorders [2]. In recent years, it has become evident that systemic inflammation is the main driver of premature atherosclerosis and its complications, together with elevated plasma levels low density lipoprotein (LDL)-cholesterol [3].

Primary hypercholesterolemia $(\mathrm{PH})$ is a lipid disorder characterized by elevated serum levels of cholesterol and low-density lipoprotein (LDL). This metabolic disorder is heterogeneous at the genetic level and includes both autosomal-dominant familial hypercholesterolemia (ADH), which has an approximate prevalence of 1:200-1:500, and the more frequent polygenic non-familial hypercholesterolemia [4]. In general, deleterious environmental factors such as a hypercholesterolemic diet and obesity are linked to the disease.

Previous studies indicate that low grade systemic inflammation is associated with $\mathrm{PH}$, which might explain the higher incidence of CVD in these patients [4,5]. In this context, several studies have shown that different soluble inflammatory markers, including tumor necrosis factor- $\alpha$ (TNF $\alpha)$, interleukin-1 (IL-1), IL-6, interferon- $\gamma$ (IFN- $\gamma$ ) and high sensitivity C reactive protein, are detected at higher levels in patients with hypercholesterolemia than in age-matched controls [5-11]. In addition, it is widely accepted that different chemotactic cytokines or chemokines are involved in the initial stage of the atherosclerotic lesion formation and more precisely in the recruitment of different leukocyte subsets [12]. In patients with hypercholesterolemia, increased circulating concentrations of monocyte chemoattractant protein-1 (MCP-1/CCL2), macrophage inflammatory protein- $1 \alpha$ (MIP-1 $\alpha / C C L 3)$, MIP-1 $\beta$ (CCL4), IL-8/CXCL8 and IFN- $\gamma$-inducible protein10 (IP-10/CXCL10) have been also described $[10,11]$. By contrast, whereas the mRNA expression of regulated on activation, normal T cell expressed and secreted (RANTES/CCL5) was reported to be increased in mononuclear cells from children with familial hypercholesteromia, this chemokine mRNA up-regulation was not found in adults with the disease [13].

Despite these findings, little is known about the different cellular components of the systemic inflammatory response present in $\mathrm{PH}$ and their clinicopathological consequences. Given that a richer understanding of immune system behavior might open new horizons for CVD prognosis and treatment, in the present study we performed an exhaustive analysis of different cellular and soluble immune players in patients with $\mathrm{PH}$ and their potential consequences in arterial leukocyte adhesion, a crucial event in atherogenesis. The activation state of platelets and relevant leukocyte subsets have been explored as well as their interactions that lead to the formation of platelet-leukocyte aggregates. Additionally, the different circulating levels of cytokines and chemokines involved in the activation state of these cellular inflammatory components or in their recruitment have been quantified and correlations with key lipid components of the disease have been established.

One of the earliest stages of atherogenesis is endothelial dysfunction, a proinflammatory and prothrombotic phenotype of the endothelium that leads to platelet activation and the adhesion and subsequent migration of $\mathrm{T}$ cells and leukocytes to the subendothelial space [14]. A correlation has been previously reported between inflammation and endothelial dysfunction in patients with $\mathrm{PH}$ [5]. Because very little is known about platelet-leukocyte-endothelium and leukocyte-endothelium interactions in $\mathrm{PH}$, we also sought to evaluate the functional significance of the inflammatory status in $\mathrm{PH}$ using an ex vivo model of dysfunctional endothelium.

\section{Materials and Methods}

\subsection{Cell culture}

Human umbilical arterial endothelial cells (HUAEC) were isolated by collagenase treatment. Details are described in the Supplemental data. 


\subsection{Human Study Populations}

A total of 43 subjects (22 $\mathrm{PH}$ patients and 21 age-matched control subjects without $\mathrm{PH}$ ) were included in the present study. Patients and control volunteers were recruited at the Endocrinology Unit at University Clinic Hospital of Valencia, Spain.

Human studies were performed following the principles outlined in the Declaration of Helsinki and were approved by the Clinical Research Ethics Committee of the University Clinical Hospital of Valencia, Spain. All patients/controls signed an informed consent to participate in the study. Further details are described in the Supplemental data.

\subsection{Flow Cytometry}

Full details are described in the Supplemental data, including the gating strategy (Figures S1-S6 and Table S2).

\subsection{Quantification of Soluble Metabolic and Inflammatory Markers}

Heparinized whole blood from patients and controls was used to quantitatively measure different soluble metabolic and inflammatory markers by Enzyme-Linked ImmunoSorbent Assay (ELISA). Further details are described in the Supplemental data.

\subsection{Leukocyte-Endothelial Cell Interactions under Flow Conditions}

Whole blood, treated or not with EDTA (Panreac, Barcelona, Spain), $10 \mathrm{mM}, 15 \mathrm{~min}, 37^{\circ} \mathrm{C}$ ), was perfused across endothelial monolayers unstimulated or stimulated with TNF $\alpha(20 \mathrm{ng} / \mathrm{mL}$, Sigma-Aldrich, Madrid, Spain), for $24 \mathrm{~h}$. Details are described in the Supplemental data.

\subsection{Immunofluorescence Studies}

Details are described in the Supplemental data.

\subsection{Statistical Analysis}

All results were analyzed using GraphPad Prism software (GraphPad Software, Inc., La Jolla, CA, USA). Values are expressed as individual data points, percentages or mean \pm standard error of the mean (SEM) when appropriate. For two-group comparisons, paired or unpaired Student's $t$ test was used in data that passed both normality (Kolmogorov-Smirnov) and equal variance (Levene) tests, as appropriate; otherwise, the non-parametric Mann Whitney $U$ test was performed. For comparisons among multiple groups, one-way analysis of variance (ANOVA) followed by post hoc Bonferroni analysis was used in data that passed both normality and equal variance tests; otherwise, the non-parametric Kruskal-Wallis test followed by Dunn's post hoc analysis was used. Data were considered statistically significant at $p<0.05$.

\section{Results}

A total of 43 subjects (22 patients with $\mathrm{PH}$ and 21 age-matched control subjects without $\mathrm{PH}$ ) were included in the present study. The demographic, clinical and laboratory characteristics of patients and controls are shown in Table 1. No statistically significant differences were found with regards to age, gender, body mass index (BMI) or waist circumference between the two groups (Table 1). By contrast, levels of total cholesterol (TC), LDL, triglycerides (TG) and apolipoprotein B (ApoB) were significantly higher in patients than in controls (Table 1). 
Table 1. Demographic and clinical features of patients and age-matched controls.

\begin{tabular}{cccc}
\hline & Control Volunteers $(\boldsymbol{n = 2 1 )}$ & PH Subjects $(\boldsymbol{n}=\mathbf{2 2})$ & $p$ Value \\
\hline Age (years) & $48.8 \pm 2.7$ & $49 \pm 3.1$ & 0.95 \\
Gender M/F (\%) & $5 / 16(23.8 / 76.2)$ & $4 / 18(18.2 / 81.8)$ & 0.72 \\
BMI (kg/m $\mathbf{2})$ & $25.4 \pm 0.7$ & $25.7 \pm 0.9$ & 0.83 \\
Waist circumference (cm) & $85.3 \pm 1.9$ & $85.7 \pm 2.2$ & 0.90 \\
SBP (mmHg) & $115.9 \pm 2.0$ & $124.7 \pm 3.6^{*}$ & $<\mathbf{0 . 0 5}$ \\
DBP (mmHg) & $71.6 \pm 1.8$ & $78.5 \pm 2.6^{*}$ & $<\mathbf{0 . 0 5}$ \\
Glucose (mg/dL) & $86.7 \pm 1.5$ & $88.1 \pm 1.9$ & 0.57 \\
TC levels (mg/dL) & $206.1 \pm 6.8$ & $264.6 \pm 8.9 * *$ & $<\mathbf{0 1}$ \\
LDL levels (mg/dL) & $130.6 \pm 5.4$ & $182.8 \pm 6.2 * *$ & $<\mathbf{0 1}$ \\
TG (mg/dL) & $80.9 \pm 7.3$ & $109.7 \pm 8.5 * *$ & $<\mathbf{0 1}$ \\
HDL levels (mg/dL) & $65.9 \pm 2.5$ & $63.4 \pm 2.9$ & 0.51 \\
ApoB (mg/dL) & $92.5 \pm 4.1$ & $127.4 \pm 5.0 * *$ & $<\mathbf{0 1}$ \\
GOT (U/L) & $21.7 \pm 0.9$ & $22.8 \pm 1.1$ & 0.42 \\
GPT (U/L) & $18.3 \pm 1.8$ & $18.5 \pm 1.1$ & 0.90 \\
Creatinine (mg/dL) & $0.7 \pm 0.0$ & $0.7 \pm 0.0$ & 0.48 \\
IgG (mg/dL) & $966.7 \pm 41.1$ & $968.5 \pm 34.4$ & 0.97 \\
Igm (mg/dL) & $100.4 \pm 7.6$ & $125.8 \pm 14.1$ & 0.14 \\
IgE total (IU/L) & $42.6 \pm 12.0$ & $50.4 \pm 16.9$ & 0.71 \\
\hline
\end{tabular}

BMI, body mass index; SBP, systolic blood pressure; DBP, diastolic blood pressure; TC, total cholesterol; LDL, low density lipoprotein; TG, triglycerides; HDL, high density lipoprotein; ApoB, apolipoprotein B; GOT, glutamic oxalacetic transaminase; GPT, glutamate-pyruvate transaminase; Ig, immunoglobulin. Data are presented as mean \pm SEM. ${ }^{*} p<0.05$ or ${ }^{* *} p<0.01$ relative to values in the control group.

\subsection{Platelet Activation Is Enhanced in Patients with PH}

We first determined the platelet activation state and levels of several mediators released upon their activation in blood samples from the two study groups using flow cytometry and ELISA. No significant differences in the number of circulating platelets were found between controls and patients (Figure 1A). By contrast, the percentage of platelets expressing PAC-1 and P-selectin (CD62P) was significantly higher in patients than in controls (Figure $1 B, C, G$ ), indicating their activation. Since P-selectin translocates to the cell surface upon cell activation, where it can be cleaved and released into the circulation as soluble P-selectin (sP-selectin), we also determined its circulating levels in plasma, finding that levels were significantly higher in the PH group than in the control group (Figure 1D). Similarly, circulating plasma levels of platelet factor-4 (PF-4/CXCL4), a platelet chemokine released upon platelet activation, were significantly higher in $\mathrm{PH}$ patients than in controls (Figure 1E). No differences, however, were encountered between $\mathrm{PH}$ patients and control subjects for the levels of circulating RANTES (regulated on activation, normal T cell expressed and secreted)//CCL5, a chemokine released by platelets and other immune cells when activated (Figure 1F). 
- Age-matched Controls

A

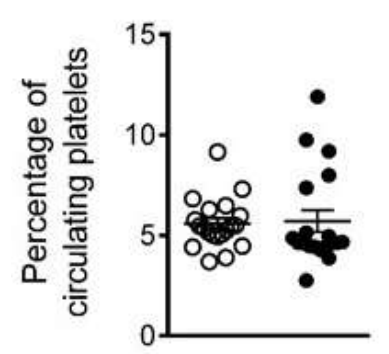

D

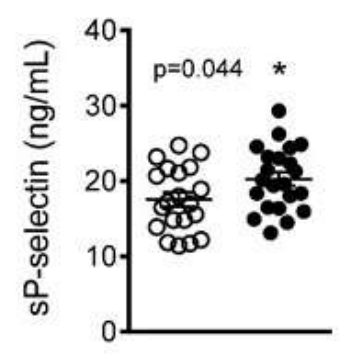

- PH Patients

B

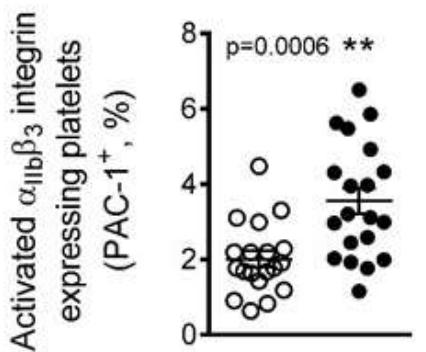

E

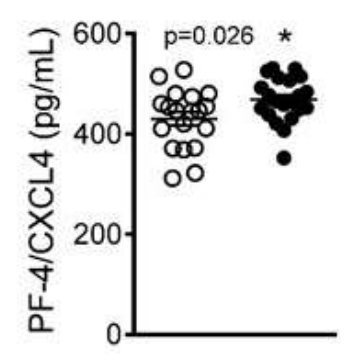

C

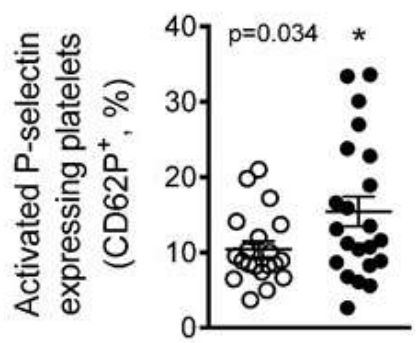

$\mathbf{F}$

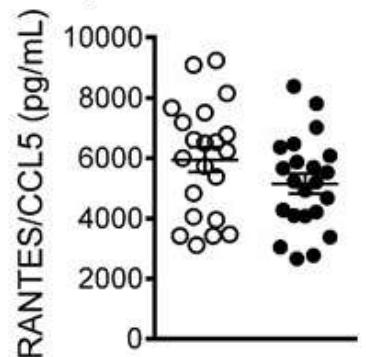

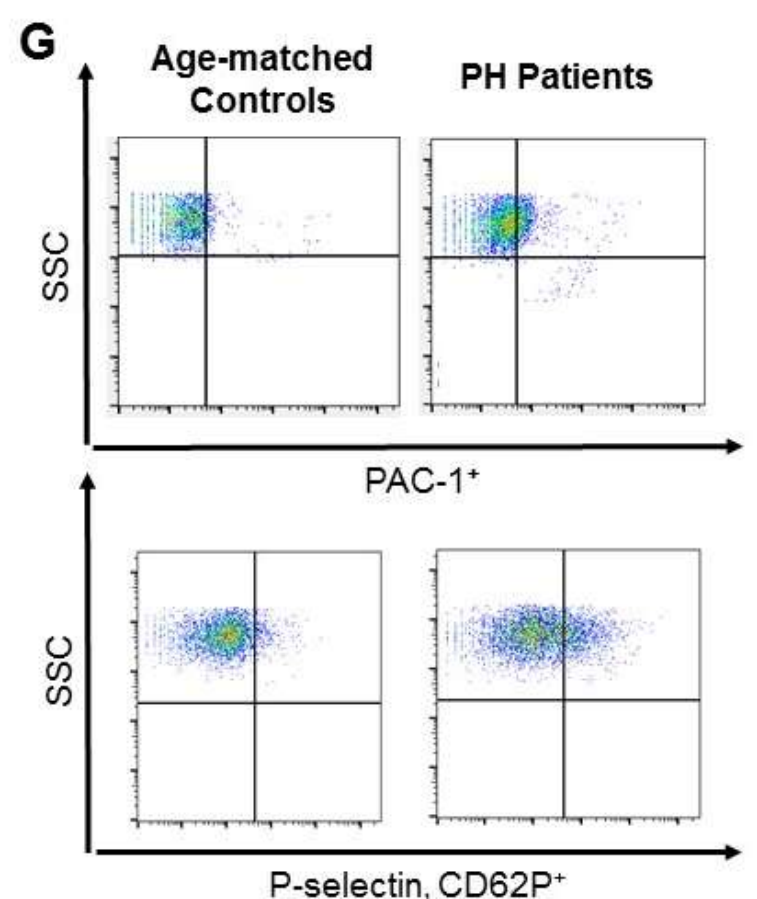

Figure 1. Platelet activation and related soluble markers are elevated in patients with $\mathrm{PH}$. Flow cytometry analysis of platelets stained with conjugated antibodies against CD41 (A), CD41 and PAC-1 (B and G), and CD41 and P-selectin (C and G). Results are expressed as percentage of positive cells. Soluble P-selectin (sP-selectin, D), PF-4/CXCL4 (E) and RANTES/CCL5 (F) plasma levels (ng or $\mathrm{pg} / \mathrm{mL}$ ) were measured by ELISA. ( $n=21$ control subjects and $n=22 \mathrm{PH}$ patients). Values are expressed as mean \pm SEM. SSC: Side Scatter. ${ }^{*} p<0.05$ or ${ }^{* *} p<0.01$ relative to values in the control group. 
3.2. The Percentage of Platelet-Neutrophil Aggregates, Activated Neutrophils, and Circulating Levels of IL-8, Are Elevated in Patients with $\mathrm{PH}$

We next evaluated several parameters related to the activation of different leukocyte subsets. No significant differences were found in the percentage of circulating neutrophils in heparinized blood between the two groups (Figure 2A); however, the percentage of platelet-neutrophil aggregates and activated neutrophils $\left(\mathrm{CD}^{+} 9^{+}\right)$was significantly higher in patients than in controls (Figure 2B,C). As some chemokines, such as growth-regulated oncogene- $\alpha$ (GRO $\alpha / C X C L 1)$ and IL-8 (CXCL8), can induce activation and chemotaxis of human neutrophils, we quantified their levels in plasma. Whereas no differences in the levels of CXCL1 were detected between the two groups (Figure 2D), plasma levels of IL-8 were significantly elevated in PH patients (Figure 2E). Of note, we found a significant association between the circulating levels of IL-8 and three clinical features of $\mathrm{PH}$ in patients: ApoB, LDL and TC (Figure 2F-H).

\section{○ Age-matched Controls}

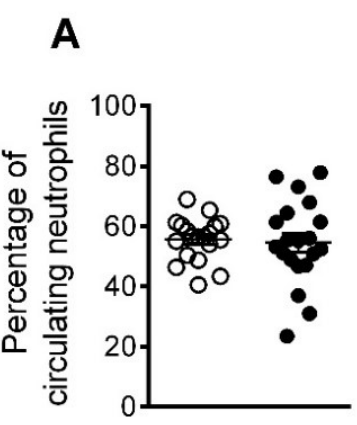

D
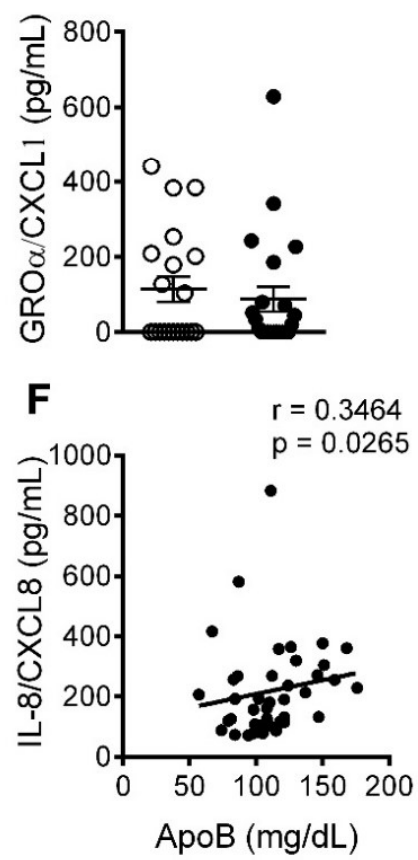

\section{- PH Patients}

B

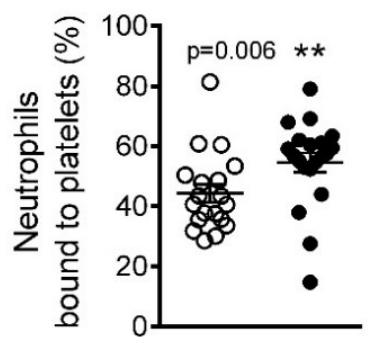

E
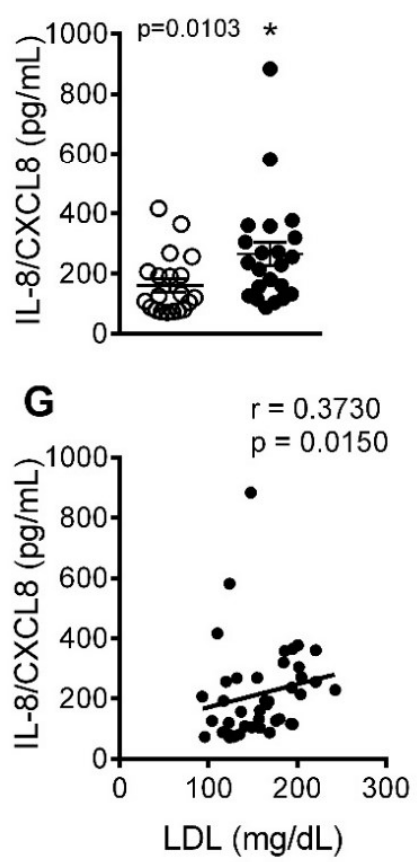
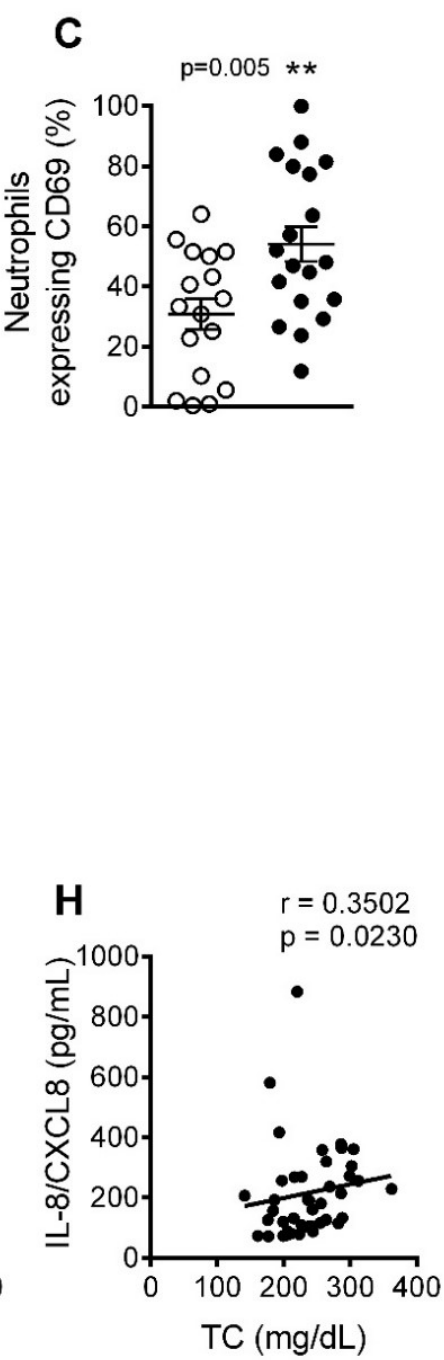

Figure 2. The percentage of platelet-neutrophil aggregates and activated neutrophils, and IL-8 circulating levels, are higher in patients with $\mathrm{PH}$. Flow cytometry analysis of heparinized whole blood co-stained with specific markers for platelets and neutrophils (A and B). Neutrophils were also stained for CD69 (C). Results are expressed as percentage of positive cells. GRO $\alpha / C X C L 1$ (D) and IL-8/CXCL8 (E) plasma levels (pg/mL) were measured by ELISA ( $n=21$ control subjects and $n=22$ $\mathrm{PH}$ patients). Values are expressed as mean \pm SEM. ${ }^{*} p<0.05$ or ${ }^{* *} p<0.01$ relative to values in the control group. Correlations between circulating IL-8 and ApoB (F), LDL (G) and TC (H) plasma levels. 
3.3. Circulating Mon 3 Monocytes, Platelet-Mon 1 and 3 Aggregates, Activated Mon 1 and 2 Monocytes, and Plasma Levels of CCL2 and $\mathrm{CX}_{3}$ CL1, Are All Elevated in Patients with PH

Three monocyte subpopulations have been described in peripheral blood based on their differential expression of the cell surface markers CD14, CD16 and CCR2 (Table S1, Supplemental data). Whereas the percentage of circulating type 1 (Mon 1) and 2 (Mon 2) monocytes in blood was not different between patients and controls, as determined by flow cytometry, we found a significantly higher percentage of circulating type 3 (Mon 3) monocytes in the former group (Figure 3A,D,G). When we analyzed platelet-monocyte aggregates, those established between platelets and Mon 1 and 3 monocytes were significantly elevated in patients with $\mathrm{PH}$ (Figure 3B,E,H). Moreover, the expression of CD11b integrin in Mon 1 and 2 monocytes, but not in Mon 3 monocytes, was significantly higher in patients than in controls, indicating their activation (Figure 3C,F,I). Analysis of the fractalkine/CX ${ }_{3} \mathrm{CL} 1$ receptor $\left(\mathrm{CX}_{3} \mathrm{CR} 1\right)$ on the different monocyte subtypes from heparinized whole blood revealed that the percentage of Mon 1 monocytes expressing this receptor was significantly higher in patients than in controls (Figure 3J). After dissociating platelets with EDTA, we found that the percentage of all monocyte subtypes positive for $\mathrm{CX}_{3} \mathrm{CR} 1$ was significantly higher in patients than in controls, with Mon 1 monocytes showing the highest percentage of $\mathrm{CX}_{3} \mathrm{CR} 1$ expression (Figure $3 \mathrm{~K}$ ). In addition, the circulating levels of MCP-1/CCL2 and soluble fractalkine/CX ${ }_{3} \mathrm{CL} 1$, ligands of CCR2 and CX ${ }_{3} \mathrm{CR} 1$ receptors respectively and involved in mononuclear cell recruitment, were significantly higher in patients (Figure $3 \mathrm{~L}, \mathrm{M}$ ), and a positive correlation was found between the circulating concentration of both chemokines and ApoB, LDL and TC levels in patients (Figure $3 \mathrm{~N}-\mathrm{S}$ ). 

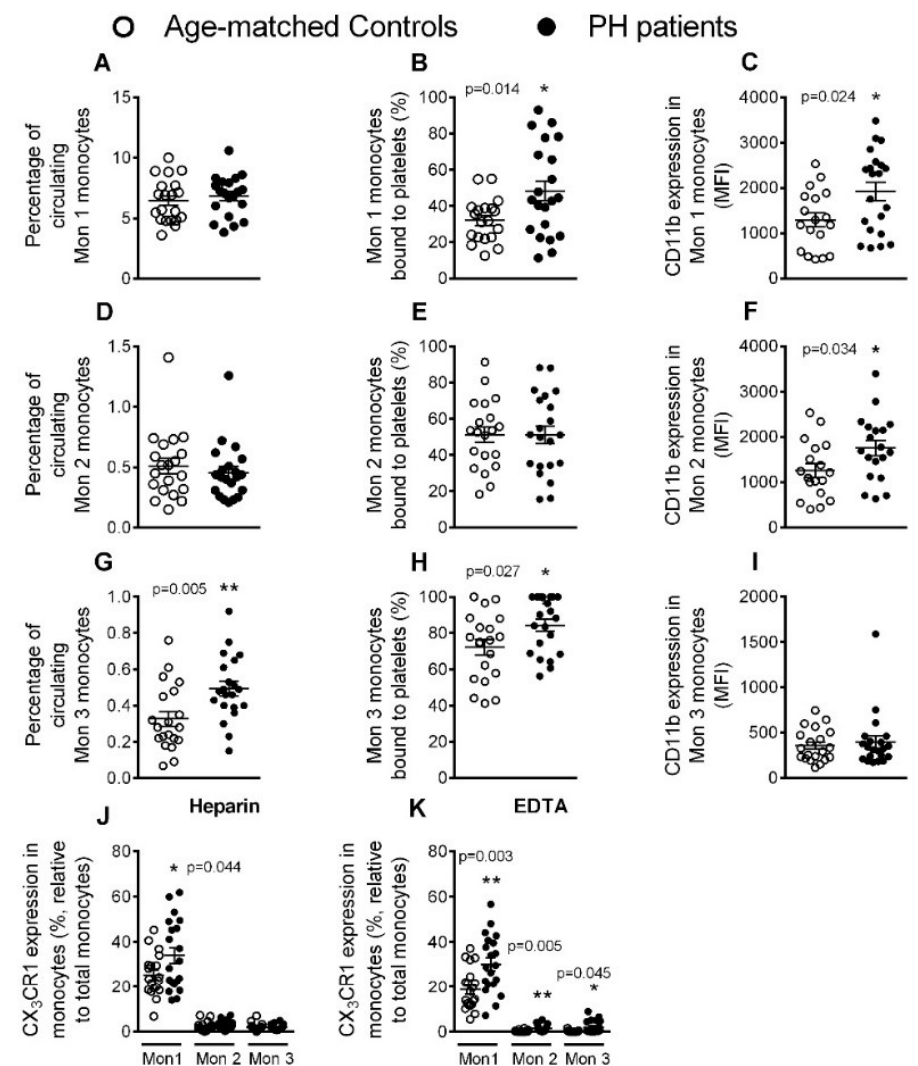

$\mathbf{L}$

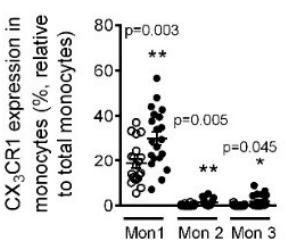

M
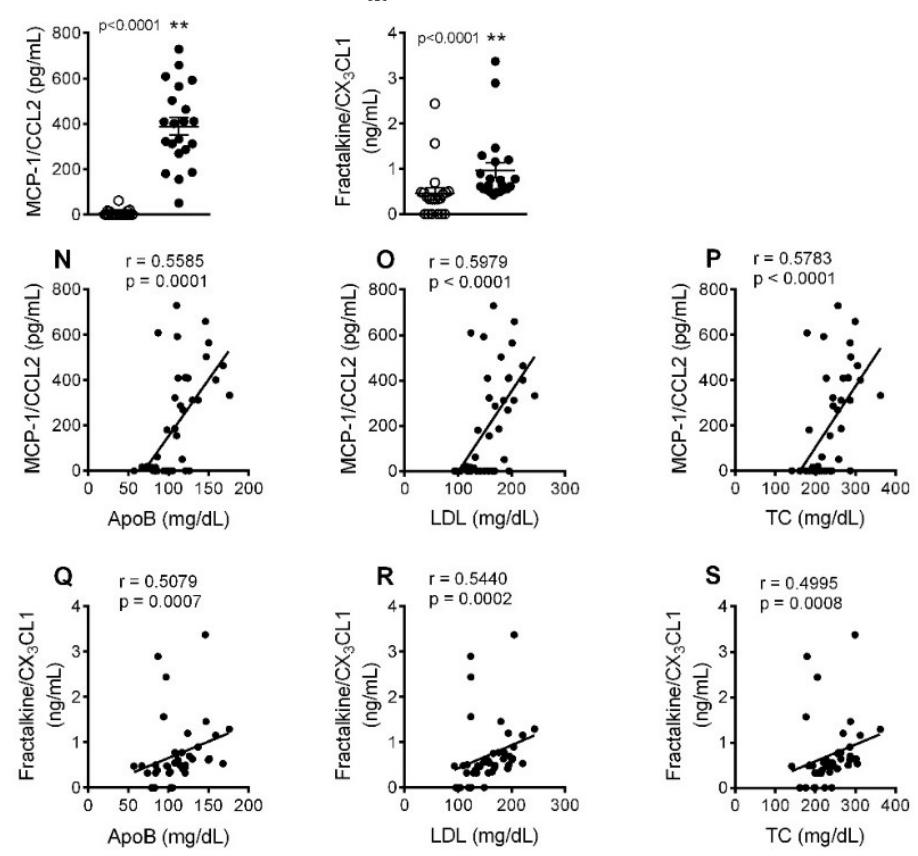

Figure 3. The percentage of circulating Mon 3 monocytes, platelet-Mon 1 and 3 aggregates, activated Mon 1 and 2 monocytes, and plasma levels of CCL2 and $\mathrm{CX}_{3} \mathrm{CL} 1$, are elevated in patients with $\mathrm{PH}$. Flow cytometry analysis of heparinized whole blood co-stained with specific markers for platelets and Mon 1, 2 and 3 monocytes (A, B, D, E, G, H), CD11b integrin $(\mathbf{C}, \mathbf{F}$ and $\mathbf{I})$, and also for $\mathrm{CX}_{3} \mathrm{CR} 1$ in heparinized and EDTA-treated whole blood $(\mathbf{J}$ and $\mathbf{K})$. Results are expressed as percentage of positive cells or mean fluorescence intensity (MFI). MCP-1/CCL2 (L) and fractalkine/CX 3 CL1 (M) plasma levels (pg or $\mathrm{ng} / \mathrm{mL}$ ) were measured by ELISA ( $n=21$ control subjects and $n=22 \mathrm{PH}$ patients). Values are expressed as mean \pm SEM. ${ }^{*} p<0.05$ or ${ }^{* *} p<0.01$ relative to values in the control group. Correlations between circulating MCP-1/CCL2 or fractalkine/CX ${ }_{3}$ CL1 and ApoB (N and $\left.\mathbf{Q}\right)$, LDL $(\mathbf{O}$ and $\mathbf{R})$ and TC (P and $\mathbf{S})$ plasma levels. 
3.4. Circulating $C D 4^{+}$Lymphocytes, Platelet-Lymphocyte $\left(C D 4^{+}\right.$and $\left.C D 8^{+}\right)$Aggregates and Lymphocyte $\left(C D 4^{+}\right.$and $\left.C D 8^{+}\right)$Activation Are Significantly increased in Patients with $\mathrm{PH}$

Mature $\mathrm{T}$ cells express the general marker $\mathrm{CD} 3$, and also express either CD4 or CD8 depending on the type of $\mathrm{T}$ cell. Whereas no differences were found in circulating numbers of $\mathrm{CD}^{+}$and $\mathrm{CD} 8^{+}$ lymphocytes between patients and controls, the number of $\mathrm{CD} 4^{+}$lymphocytes was higher the former group (Figure 4A,D,J) and positively correlated with ApoB, LDL and TC levels (Figure 4G-I). Moreover, the percentage of $\mathrm{CD}^{+}, \mathrm{CD}^{+}$and $\mathrm{CD} 8^{+}$lymphocytes bound to platelets, and also their activation state, $\left(\mathrm{CD} 9^{+}\right)$was greater in patients than in control subjects (Figure $\left.4 \mathrm{~B}, \mathrm{C}, \mathrm{E}, \mathrm{F}, \mathrm{K}, \mathrm{L}\right)$. Interestingly, a positive correlation was found between the percentage of $\mathrm{CD} 69^{+} \mathrm{CD} 8^{+}$cells and the lipid profile (ApoB, LDL and TC levels) (Figure 4M-O).

Closer inspection of the different $\mathrm{CD} 4^{+} \mathrm{T}$ lymphocyte subtypes revealed an increased number of circulating Th2 and Th17, but not Th1, lymphocytes in patients (Figure 5A,D,G). Furthermore, we found that the percentage of circulating platelet-Th lymphocyte aggregates of all three subtypes, as well as their activation state $\left(\mathrm{CD}^{2} 9^{+}\right)$, was higher in patients than in controls (Figure 5B,C,E,F,H,I). In contrast to $\mathrm{CD}^{+}$cells, the percentage of circulating Treg lymphocytes (Figure 5J), as well as the Treg/Th17 ratio (Figure 5L), was significantly lower in patients. However, no differences were found between patients and controls in the percentage of circulating Treg lymphocyte-platelet aggregates (Figure 5K). Of note, whereas the circulating levels of IL-12, a cytokine involved in the differentiation of naïve T cells to Th1 cells, were significantly elevated in patients, plasma levels of IFN $\gamma$, a cytokine released by Th1 lymphocytes, were not different from those of control subjects (Figure $5 \mathrm{M}, \mathrm{N}$ ). By contrast, levels of the anti-inflammatory cytokines IL-4 and IL-10, which are mainly produced by Th2 and Treg lymphocytes, respectively, were significantly lower in the circulation of $\mathrm{PH}$ patients (Figure 5O,P). Indeed, an inverse correlation was found between IL-4 and IL-10 and the lipid profile associated with PH (Figure 5Q-V). 


\section{○ Age-matched Controls • PH Patients}

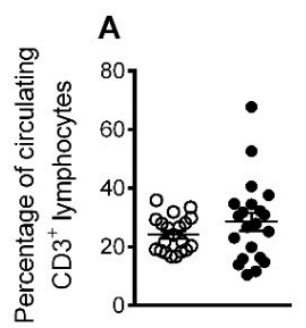

D
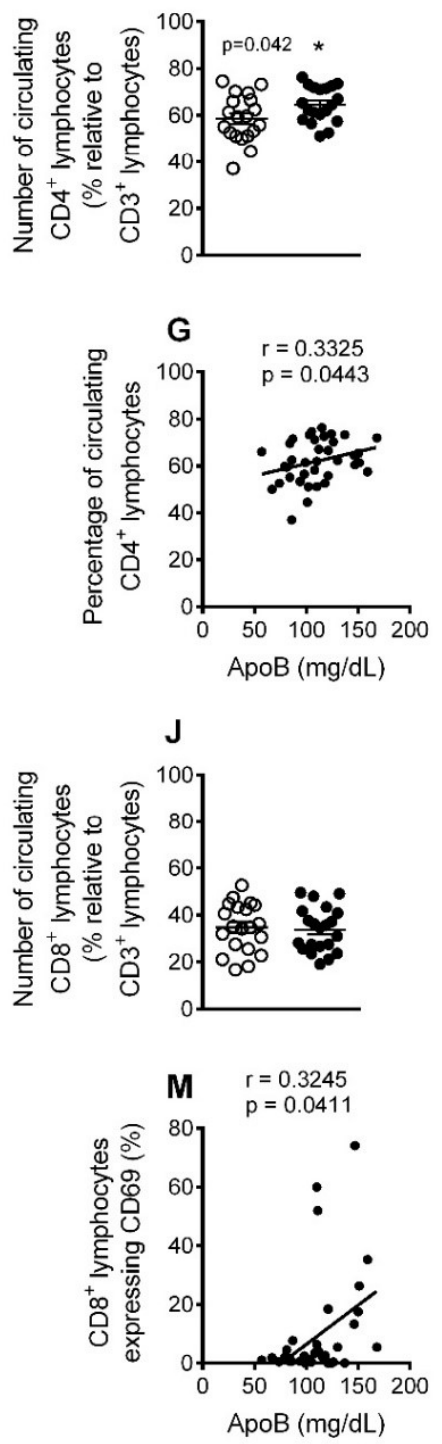

B

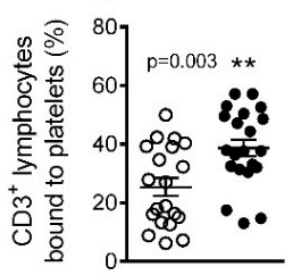

$E$
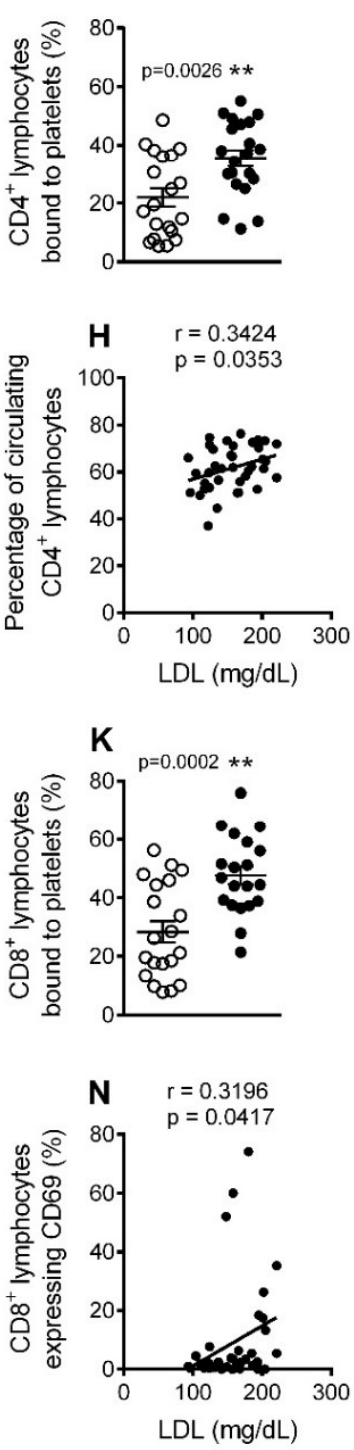
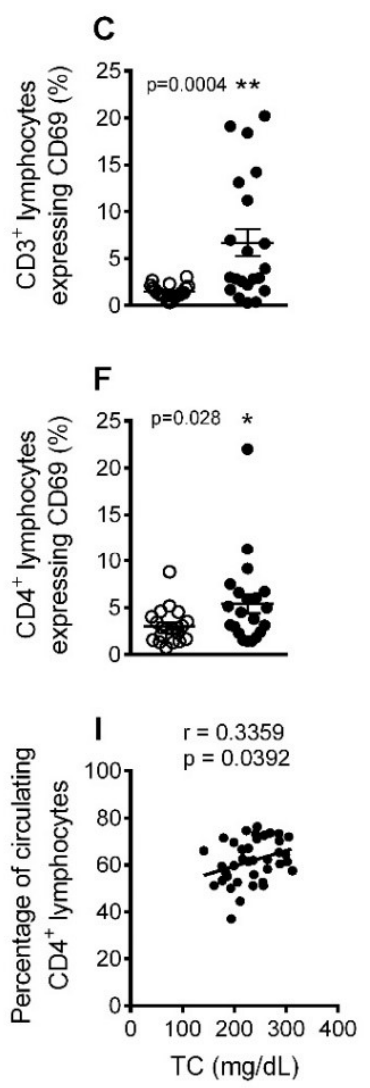

L
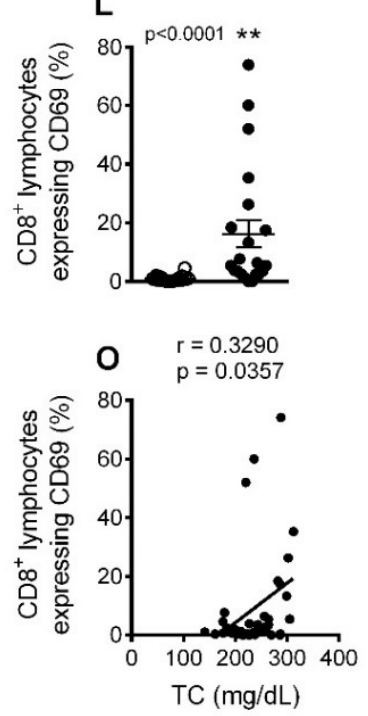

Figure 4. The percentage of circulating $\mathrm{CD} 4^{+}$lymphocytes, platelet-lymphocyte $\left(\mathrm{CD} 4^{+}\right.$and $\left.\mathrm{CD} 8^{+}\right)$ aggregates, and lymphocyte $\left(\mathrm{CD} 4^{+}\right.$and $\left.\mathrm{CD} 8^{+}\right)$activation, are significantly elevated in patients with $\mathrm{PH}$. Heparinized whole blood was co-stained with specific markers for platelets and $\mathrm{CD}^{+}, \mathrm{CD}^{+}$and CD8 ${ }^{+}$lymphocytes (A, B, D, E, J and K) as well as for CD69 (C, F and L). Results are expressed as the percentage of positive cells ( $n=21$ control subjects and $n=22 \mathrm{PH}$ patients). Values are expressed as mean \pm SEM. ${ }^{*} p<0.05$ or ${ }^{* *} p<0.01$ relative to values in the control group. Correlations between circulating $\mathrm{CD}^{+}$cells and activated $\mathrm{CD} 8^{+}$lymphocytes and ApoB (G and $\left.\mathbf{M}\right), \mathrm{LDL}(\mathbf{H}$ and $\mathbf{N})$ and TC (I and $\mathbf{O})$ plasma levels. 
O Age-matched Controls
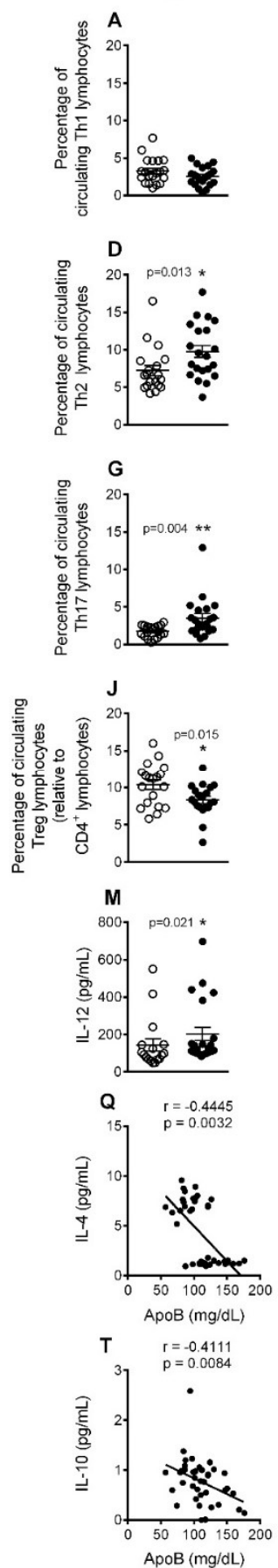
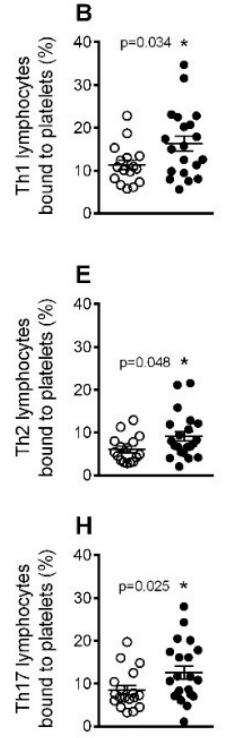

K
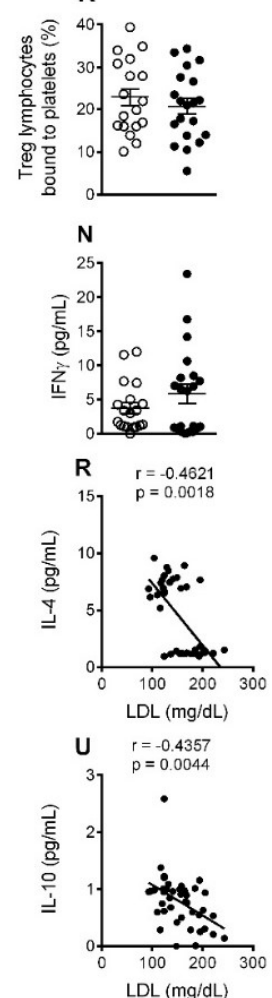

PH Patients
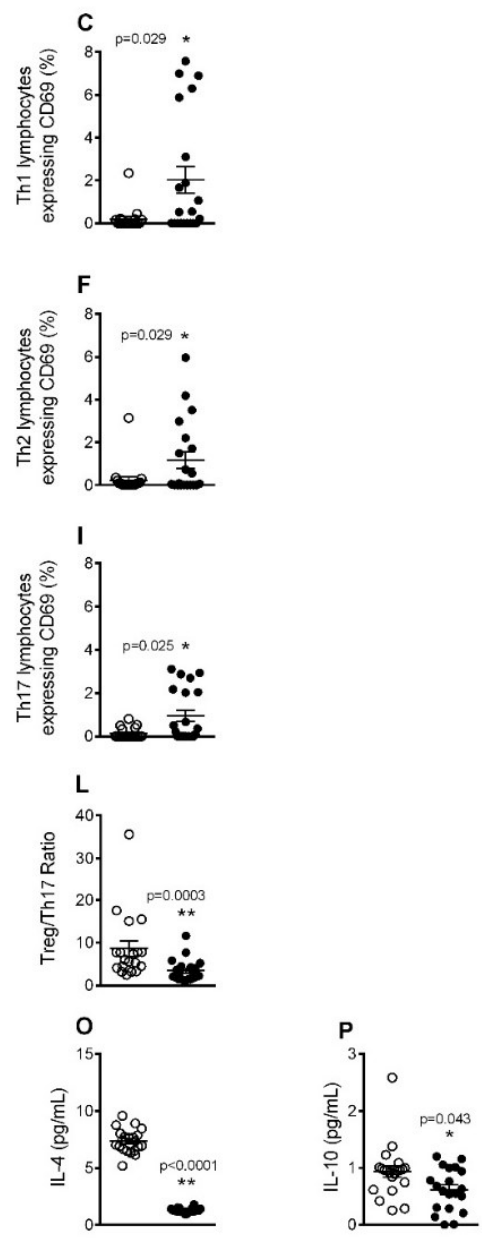

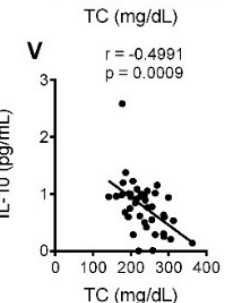

Figure 5. The percentage of circulating Th2 and Th17 lymphocytes, platelet-lymphocyte aggregates, lymphocyte activation and IL-12 circulating levels, are significantly increased in patients with $\mathrm{PH}$, whereas the percentage of circulating Treg cells, Treg/Th17 ratio, and IL-4 and IL-10 plasma levels, are decreased. Heparinized whole blood was co-stained with specific markers for platelets and Th1, Th2, Th17 and Treg lymphocytes (A, B, D, E, G, H, J and K) as well as for CD69 (C, F and I). Treg/Th17 ratio was also determined (L). Results are expressed as percentage of positive cells. IL-12 (M), IFN $\gamma$ (N), IL-4 (O) and IL-10 (P) plasma levels (pg/mL) were measured by ELISA $(n=21$ control subjects and $n=22 \mathrm{PH}$ patients). Values are expressed as mean \pm SEM. ${ }^{*} p<0.05$ or ${ }^{* *} p<0.01$ relative to values in the control group. Correlations between circulating IL-4 or IL-10 and ApoB ( $\mathbf{Q}$ and $\mathbf{T}), \mathrm{LDL}$ ( $\mathbf{R}$ and $\mathbf{U})$ and TC (S and V) plasma levels. 


\subsection{Circulating Levels of Pro-Inflammatory Cytokines but not Adipokines Are Increased in PH Patients}

Th17 cells produce TNF $\alpha$ and IL-6 [15], and an increase in the plasma levels of these pro-inflammatory cytokines has been reported in patients with $\mathrm{PH}[6,7,9]$. We noted similar findings in our patient cohort (Figure 6A,B); moreover, a positive association was found between IL-6 plasma levels and the levels of circulating ApoB, LDL and TC (Figure 6C-E). By contrast, no differences were found for the circulating levels of adiponectin, leptin or ghrelin between patients and controls (Figure 6F-H).

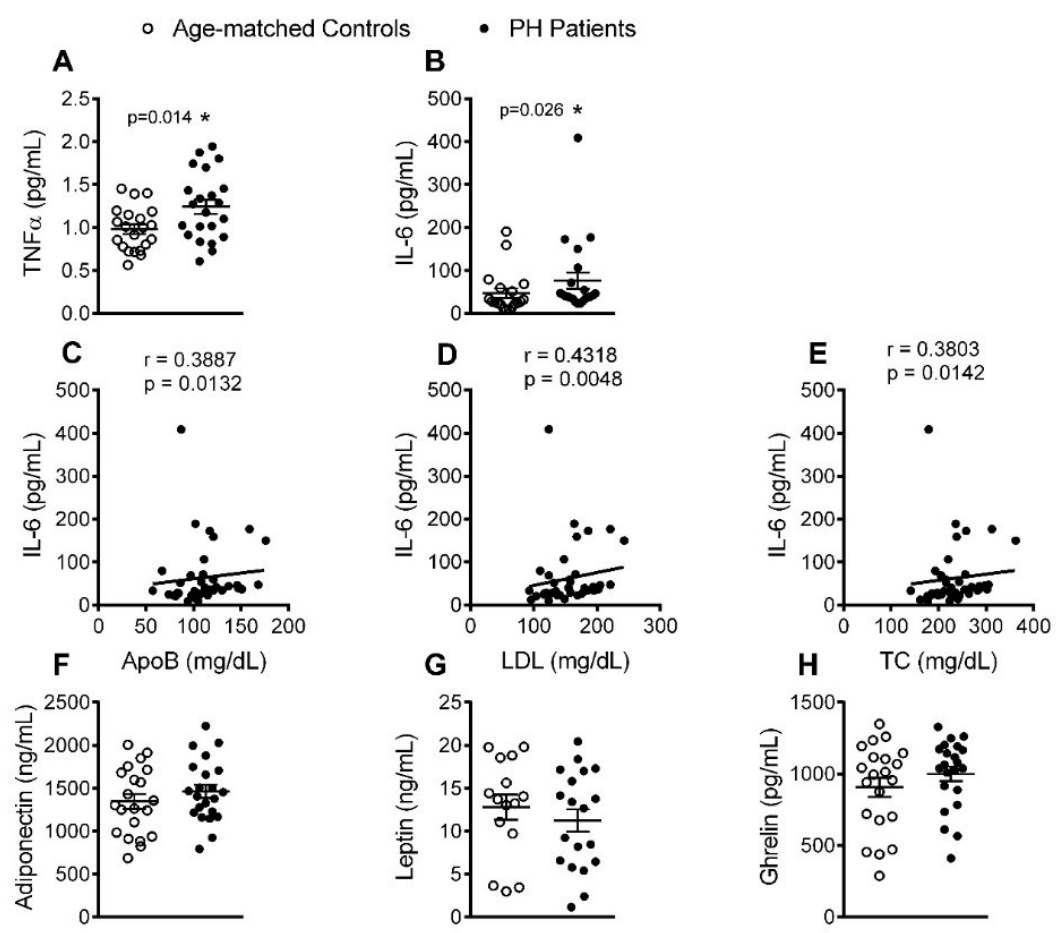

Figure 6. Increased circulating levels of pro-inflammatory cytokines but not adipokines in patients with PH. TNF $\alpha(\mathbf{A})$, IL-6 (B), adiponectin (F), leptin (G) and ghrelin (H) plasma levels (pg or ng/mL) were measured by ELISA ( $n=21$ control subjects and $n=22 \mathrm{PH}$ patients). Values are expressed as mean \pm SEM. ${ }^{*} p<0.05$ or ${ }^{* *} p<0.01$ relative to values in the control group. Correlations between circulating IL-6 and ApoB (C), LDL (D) and TC (E) plasma levels.

3.6. Circulating Platelet-Leukocytes and Leukocytes from PH Patients Have Increased Adhesiveness to TNF $\alpha$-Stimulated HUAEC

Endothelial dysfunction is one of the earliest stages of atherogenesis, and leads to the adhesion and the subsequent migration of leukocytes [14]. Because TNF $\alpha$ is a central cytokine/adipokine in hypercholesterolemia $[9,11]$, we next explored the functional consequences of the elevated levels of $\mathrm{TNF} \alpha$ in patients with PH. We first examined the adhesion of platelet-leukocyte aggregates and leukocytes alone to unstimulated or TNF $\alpha$-stimulated arterial endothelial cells (HUAEC) under dynamic flow conditions. To do this, experiments were carried out with heparinized or EDTA-treated blood, which promotes the dissociation of platelets from leukocytes.

When heparinized, diluted whole blood from patients and controls was perfused across unstimulated HUAEC, leukocyte adhesiveness was significantly greater in the PH group (Figure 7A). After exposure of HUAEC to TNF $\alpha$ for $24 \mathrm{~h}$, leukocyte adhesiveness increased in both groups and remained significantly greater in the PH group (Figure 7A). Importantly, when platelets were disaggregated from leukocytes with EDTA, leukocyte adhesion was still significantly greater in the $\mathrm{PH}$ group than the control group (Figure 7B), despite the significant decrease in the number of adhered leukocytes to stimulated HUAEC after platelet disaggregation (Figure 7A,B). In agreement 
with these observations, immunofluorescence studies revealed enhanced adherent platelet-leukocyte complexes to $\mathrm{TNF} \alpha$-stimulated endothelial cells from PH patients compared with age-matched controls (Figure 7C,D). Furthermore, when platelets were disaggregated from leukocytes with EDTA, leukocyte adhesion to TNF $\alpha$-stimulated HUAEC was notably diminished but this parameter was markedly greater in the $\mathrm{PH}$ group than the control group (Figure 7E,F).

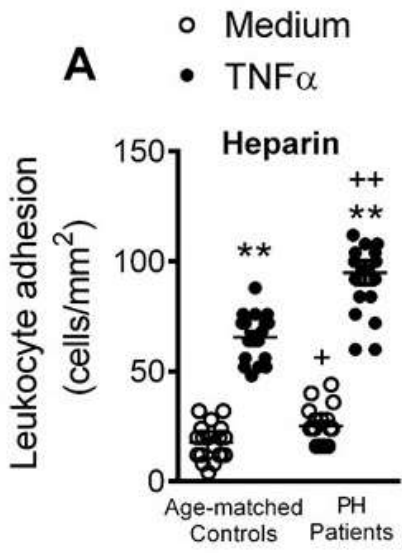

B
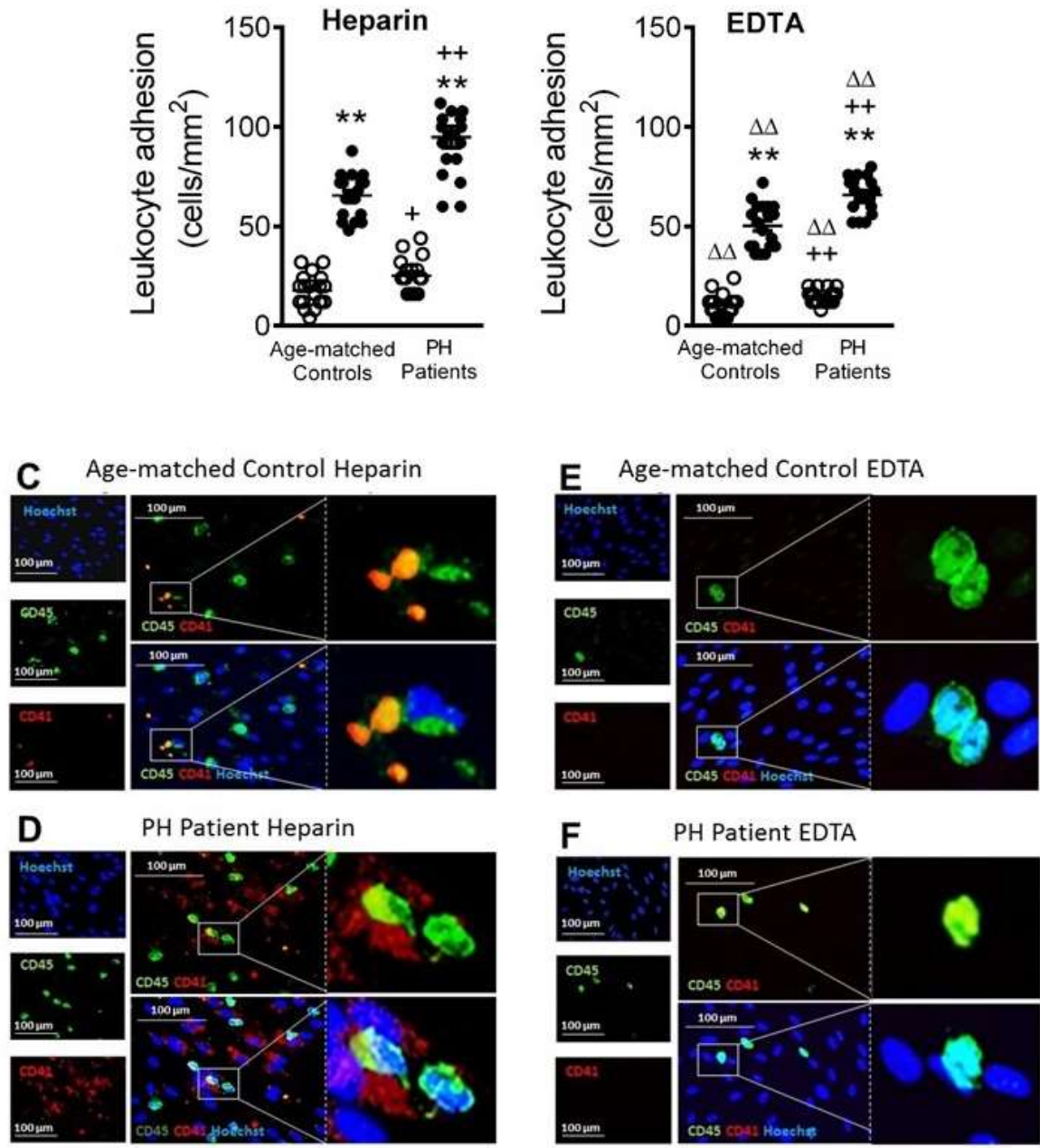

Figure 7. Circulating platelet-leukocyte aggregates and leukocytes from $\mathrm{PH}$ patients show increased adhesiveness to TNF $\alpha$-stimulated HUAEC. HUAEC were stimulated or not with TNF $\alpha(20 \mathrm{ng} / \mathrm{mL})$ for $24 \mathrm{~h}$. Subsequently, whole blood from patients and controls, incubated without (A) or with EDTA (B), was perfused across endothelial monolayers for $7 \mathrm{~min}$ at $0.5 \mathrm{dyn} / \mathrm{cm}^{2}$ and leukocyte adhesion quantified (cells $\left./ \mathrm{mm}^{2}\right)$. Values are expressed as mean \pm SEM $(n=21$ control subjects and $n=22$ PH patients). ${ }^{* *} p<0.01$ relative to values in the medium group; $+p<0.05$ or $++p<0.01$ relative to respective values in the control group; $\Delta \Delta p<0.01$ relative to respective values in the heparin group. Immunofluorescence analysis showing adherent platelet-leukocyte cell complexes to TNF $\alpha$-stimulated HUAEC (C-F). Heparinized blood from age-matched controls and patients with PH was incubated without or with EDTA. After the flow chamber assay, cells were fixed with $4 \%$ paraformaldehyde and blocked in phosphate-buffered saline (PBS) containing 1\% bovine serum albumin (BSA). Then, cells were incubated for $2 \mathrm{~h}$ with an Alexa 488-conjugated antibody against human CD45 (1:50 dilution, green) and an allophycocyanin (APC)-conjugated antibody against human CD41 (1:50 dilution, red). Nuclei of endothelial cells and leukocytes were stained with Hoechst (blue). Images were captured with a Zeis Axio Observer A1 fluorescence microscope. 


\section{Discussion}

$\mathrm{PH}$ is associated with risk of developing arteriosclerosis and the likelihood of future serious ischemic events. Previous studies have provided evidence of low systemic inflammation in patients with hypercholesterolemia $[6,7,9-11,16]$. Here, we carried out a detailed characterization of different immune players and soluble inflammatory markers in $\mathrm{PH}$ and correlated these data with the circulating levels of key lipid components. The enhanced inflammatory status of PH reported herein has functional consequences, as illustrated for circulating platelet-bound leukocytes, which have increased adhesiveness to dysfunctional arterial endothelium, a prominent feature of the atherogenic process.

Platelet activation is known to be associated with atherogenesis and cardiovascular morbidity [17]. Indeed, upon their activation, platelets express specific cell adhesion molecules such as P-selectin, and release several inflammatory chemokines including PF-4/CXCL4 or RANTES/CCL5 [17]. We show that patients with $\mathrm{PH}$ present a pro-thrombotic state characterized by increased platelet activation (P-selectin ${ }^{+}$and PAC- $1^{+}$platelets). While hypercholesterolemia has been previously associated with platelet activation $[5,16]$, we found that patients have both increased circulating levels of sP-selectin and PF-4/CXCL4, which are involved in multiple atherogenic processes. Indeed, different platelet surface molecules such as GPIIb/IIIa (recognized by PAC-1) or P-selectin are critically involved in the interaction of platelets to endothelial cells and leukocytes [17], all of which are central for atherosclerotic lesion formation.

To gain insight into the immune state of the hypercholesterolemic environment of $\mathrm{PH}$, we examined different leukocyte subtypes. An increase in leukocyte activation in vitro has been reported in subjects at high cardiovascular risk (hyperlipidemia) [18]. In our study, whereas no differences in the percentage of circulating neutrophils were detected between patients and controls, a clear increase in the percentage of activated cells $\left(\mathrm{CD} 9^{+}\right)$was observed, suggesting the existence of a proatherogenic state. This is consistent with our finding of increased circulating levels of CXCL8, which is involved in neutrophil activation, in the PH group, as has been reported previously, albeit in patients with $\mathrm{FH}$ [10]. Also, the plasma concentrations of this chemokine positively correlated with the circulating levels of key lipids in PH, ApoB, LDL and TC. Overall, these results indicate that IL-8 might have utility as biomarker of atherosclerotic risk in $\mathrm{PH}$.

Human monocytes are a heterogeneous cell population that are commonly classified into three subtypes: classical CD14 ${ }^{+} \mathrm{CD} 16^{-} \mathrm{CCR} 2^{+}$(Mon 1), intermediate CD14 ${ }^{+} \mathrm{CD} 16^{+} \mathrm{CCR} 2^{+}$(Mon 2), and nonclassical CD14 ${ }^{+} \mathrm{CD} 16^{+} \mathrm{CCR} 2^{-}$(Mon 3) [19]. There is evidence to support that adults with FH have a pro-inflammatory imbalance in circulating monocyte subpopulations (Mon 1) [20], although another study indicated that the levels of Mon 2 and/or Mon 3 subtypes were increased in hyperlipidemia and associated with atherosclerosis development [21]. We found that only the percentage of the nonclassical/Mon 3 subtype was increased in patients over controls, and this positively correlated with the circulating levels of apoB, LDL and TC (Figure S7, Supplemental data). By contrast, Mon 1 and Mon 2 subtypes, both of which express the CCR2 receptor, were significantly activated in patients. We also show for the first time an increase in the percentage of fractalkine $/ \mathrm{CX}_{3} \mathrm{CL} 1$ receptor $\left(\mathrm{CX}_{3} \mathrm{CR} 1\right)$ expression on Mon 1 monocytes in heparinized whole blood, and on all monocyte subsets when platelets were dissociated. In line with these observations, studies of atherosclerosis in mice suggest that both inflammatory (similar to human Mon 1) and patrolling (similar to human Mon 3) monocytes are involved in disease progression [22]. In humans, different studies have noted increases in circulating $\mathrm{CD} 16^{+}$monocytes in cardiovascular disease [22], which are possibly linked to disease outcome [23]. There is also evidence to support that mobilized classical monocytes from the bone marrow mature into nonclassical monocytes through an intermediate subset. How these different monocyte subtypes correlate with disease pathogenesis and clinical outcomes in $\mathrm{PH}$ is, however, unknown. Nevertheless, it is likely that those subtypes expressing both CCR2 and $\mathrm{CX}_{3} \mathrm{CR} 1$ are more prone to migrate from the circulation into arterial walls through the interaction with their cognate ligands CCL2 and $\mathrm{CX}_{3} \mathrm{CL} 1$, the circulating levels of which were significantly elevated in patients and correlated positively with plasma ApoB, LDL and TC content. 
To the best of our knowledge, the associations we found between T lymphocytes and PH have not previously been reported. Four findings are worthy of mention. First, the percentage of circulating $\mathrm{CD} 4^{+}$cells was significantly higher in patients with $\mathrm{PH}$ than controls, and directly correlated with levels of ApoB, LDL and TC. Of note, this increase was likely due to the increased numbers of circulating Th2 and Th17 cells. Second, most of the T cell subpopulations in patients displayed an activated state and positive correlations were found between the percentage of $\mathrm{CD} 8^{+} \mathrm{CD} 69^{+}$cells and key lipid features of the disease. Third, the percentage of circulating Treg cells and the Treg/Th17 ratio was decreased in PH patients. Finally, whereas IL-12, TNF $\alpha$ and IL-6 plasma levels were increased in patients, levels of the anti-inflammatory cytokines IL-4 or IL-10 were decreased and inversely correlated with the levels of ApoB, LDL and TC. These observations, overall, link the cellular and molecular inflammatory profile to a possible pro-atherogenic enviroment. Along this line, it is well known that both $\mathrm{CD} 4^{+}$ and $\mathrm{CD} 8^{+} \mathrm{T}$ cells are involved in atherosclerosis development [24]. While the role of Th2 cells in atherogenesis remains debated [24], it has recently been shown that patients with coronary artery atherosclerosis had an impaired Treg/Th17 ratio together with reduced serum levels of IL-10 [25]. Th17 cells repress the function of Treg cells, contributing to an inflammatory milieu. Moreover, whereas Th17 cells can produce the inflammatory cytokines TNF $\alpha$ and IL-6 [15], Treg cells generate and release the anti-inflammatory cytokine IL-10. It is therefore tempting to speculate that there is a conversion of Treg cells into Th17 cells in PH. Finally, although IL-4 is a classic Th2 cytokine, the decreased levels found in patients suggest an alternative cellular origin of this cytokine. Indeed, potential sources of IL-4 are double-positive CD4/CD8 lymphocytes, basophils or natural killer T cells [26,27] whose circulating levels may be decreased in this pathology, although this requires further investigation.

We used a dynamic flow chamber model to explore the functional consequences of platelet-leukocyte-endothelium (heparin) or leukocyte-endothelium (EDTA) interactions, finding that adhesion of platelet-leukocyte aggregates to HUAEC, stimulated or not with $\mathrm{TNF} \alpha$, was significantly higher in the patient group. The increased adhesion to functional (nonstimulated) endothelium was likely due to neutrophil and monocyte activation and consequent over-expression of CD11b/CD18 integrin, which interacts with the constitutively expressed intercellular cell adhesion molecule- 1 in endothelium. Furthermore, platelets seem to be critical for leukocyte adhesion to dysfunctional (stimulated) arterial endothelium, as leukocyte-endothelium interactions were significantly impaired when platelets were dissociated with EDTA. It is widely accepted that activated platelets can mediate the endothelial adhesion of circulating leukocytes, a characteristic feature of the dysfunctional endothelium [14,28-31]. We also found a significant enhancement in the percentage of platelet-leukocyte aggregates, which were established with almost all the leukocyte subsets investigated in a background of $\mathrm{PH}$. The increased number of these aggregates have been detected in the peripheral circulation of patients with unstable angina or other coronary diseases, and they have been considered a predictive factor of acute myocardial infarction [32]. This platelet-leukocyte interaction is in all probability due to the interaction of platelet P-selectin with its ligand, P-selectin glycoprotein ligand-1, present on leukocyte surfaces, which in turns facilitates the interaction between these aggregates and dysfunctional endothelium, a key event in arteries prone to arteriosclerotic lesion development [14].

In conclusion, we report that the low grade systemic inflammation associated with $\mathrm{PH}$ is accompanied by a pro-thrombotic state with heightened platelet activation and associated circulating soluble markers. This platelet activation state in $\mathrm{PH}$, together with the activation of different leukocyte subsets, results in the formation of platelet-leukocyte aggregates and their adhesion to dysfunctional arterial endothelium, suggesting a potential link between systemic inflammation and CVD development in this metabolic disorder. Finally, the positive correlations between key lipid features of PH and different circulating inflammatory mediators (IL-8, MCP-1, fractalkine or IL-6) and the negative correlations between these lipids and anti-inflammatory cytokines (IL-4 and IL-10) might be used as potential markers of CVD. Overall, the modulation of the cellular and molecular 
inflammatory components in $\mathrm{PH}$, as well as the lipid profile, might be crucial to prevent further cardiovascular complications.

Supplementary Materials: The following are available online at http:/ /www.mdpi.com/2077-0383/8/1/18/s1, Figure S1. Gating strategy for human platelets in whole blood according to morphological properties and CD41 detection by flow cytometry, Figure S2. Gating strategy for human neutrophils in whole blood according to morphological properties and CD16 expression by flow cytometry, Figure S3. Gating strategy for human monocyte detection in whole blood by flow cytometry, Figure S4. Gating strategy for human T lymphocyte detection in whole blood by flow cytometry, Figure S5. Gating strategy for human T helper lymphocyte detection in whole blood by flow cytometry, Figure S6. Gating strategy for human regulatory T lymphocyte (Treg) detection in whole blood by flow cytometry, Figure S7. Positive correlation between the percentage of nonclassical/Mon 3 monocytes and the circulating levels of ApoB (A), LDL (B) and TC (C). Table S1. Differential markers of monocyte subpopulations, Table S2. Differential markers for detection of Th lymphocyte subpopulations.

Author Contributions: J.T.R., J.F.A. and M.-J.S. contributed to the conception and design of the study; A.C., P.M., E.D., E.P., S.M.-H., L.P., J.T.R., J.F.A. and M.-J.S. acquired, analyzed and interpreted the data; A.C., P.M., E.D., J.F.A. and M.-J.S. wrote the manuscript and S.M.-H., L.P. and J.T.R. revised it critically. All the authors have read and approved the submission of the manuscript.

Funding: This work was supported by the Spanish Ministry of Economy and Competiveness (grant numbers SAF2014-57845-R, SAF2017-89714-R); Carlos III Health Institute and the European Regional Development Fund (grant numbers PI15/00082, PIE15/00013, PI18/00209).

Acknowledgments: PM acknowledge pre-doctoral funding from the Spanish Ministry of Innovation and Competitiveness (FPI) and AC and ED to the Generalitat Valenciana.

Conflicts of Interest: The authors declare no conflict of interest.

\section{References}

1. Lozano, R.; Naghavi, M.; Foreman, K.; Lim, S.; Shibuya, K.; Aboyans, V.; Abraham, J.; Adair, T.; Aggarwal, R.; Ahn, S.Y.; et al. Global and regional mortality from 235 causes of death for 20 age groups in 1990 and 2010: A systematic analysis for the Global Burden of Disease Study 2010. Lancet 2012, 380, 2095-2128. [CrossRef]

2. Ross, R. The pathogenesis of atherosclerosis: A perspective for the 1990s. Nature 1993, 362, 801-809. [CrossRef] [PubMed]

3. Catapano, A.L.; Pirillo, A.; Norata, G.D. Vascular inflammation and low-density lipoproteins: Is cholesterol the link? A lesson from the clinical trials. Br. J. Pharmacol. 2017, 174, 3973-3985. [CrossRef] [PubMed]

4. Langslet, G.; Emery, M.; Wasserman, S.M. Evolocumab (AMG 145) for primary hypercholesterolemia. Expert Rev. Cardiovasc. Ther. 2015, 13, 477-488. [CrossRef] [PubMed]

5. Barale, C.; Frascaroli, C.; Senkeev, R.; Cavalot, F.; Russo, I. Simvastatin Effects on Inflammation and Platelet Activation Markers in Hypercholesterolemia. Biomed Res. Int. 2018, 2018, 6508709. [CrossRef] [PubMed]

6. Real, J.T.; Martinez-Hervas, S.; Garcia-Garcia, A.B.; Civera, M.; Pallardo, F.V.; Ascaso, J.F.; Vina, J.R.; Chaves, F.J.; Carmena, R. Circulating mononuclear cells nuclear factor-kappa B activity, plasma xanthine oxidase, and low grade inflammatory markers in adult patients with familial hypercholesterolaemia. Eur. J. Clin. Investig. 2010, 40, 89-94. [CrossRef] [PubMed]

7. Holven, K.B.; Narverud, I.; Lindvig, H.W.; Halvorsen, B.; Langslet, G.; Nenseter, M.S.; Ulven, S.M.; Ose, L.; Aukrust, P.; Retterstol, K. Subjects with familial hypercholesterolemia are characterized by an inflammatory phenotype despite long-term intensive cholesterol lowering treatment. Atherosclerosis 2014, 233, 561-567. [CrossRef] [PubMed]

8. Cheng, H.M.; Ye, Z.X.; Chiou, K.R.; Lin, S.J.; Charng, M.J. Vascular stiffness in familial hypercholesterolaemia is associated with C-reactive protein and cholesterol burden. Eur. J. Clin. Investig. 2007, 37, 197-206. [CrossRef] [PubMed]

9. Sampietro, T.; Tuoni, M.; Ferdeghini, M.; Ciardi, A.; Marraccini, P.; Prontera, C.; Sassi, G.; Taddei, M.; Bionda, A. Plasma cholesterol regulates soluble cell adhesion molecule expression in familial hypercholesterolemia. Circulation 1997, 96, 1381-1385. [CrossRef]

10. Cortes, R.; Ivorra, C.; Martinez-Hervas, S.; Pedro, T.; Gonzalez-Albert, V.; Artero, A.; Adam, V.; Garcia-Garcia, A.B.; Ascaso, J.F.; Real, J.T.; et al. Postprandial Changes in Chemokines Related to Early Atherosclerotic Processes in Familial Hypercholesterolemic Subjects: A Preliminary Study. Arch. Med. Res. 2016, 47, 33-39. [CrossRef] 
11. Hansen, M.; Kuhlman, A.C.B.; Sahl, R.E.; Kelly, B.; Morville, T.; Dohlmann, T.L.; Chrois, K.M.; Larsen, S.; Helge, J.W.; Dela, F. Inflammatory biomarkers in patients in Simvastatin treatment: No effect of co-enzyme Q10 supplementation. Cytokine 2019, 113, 393-399. [CrossRef] [PubMed]

12. Charo, I.F.; Ransohoff, R.M. The many roles of chemokines and chemokine receptors in inflammation. N. Engl. J. Med. 2006, 354, 610-621. [CrossRef] [PubMed]

13. Holven, K.B.; Damas, J.K.; Yndestad, A.; Waehre, T.; Ueland, T.; Halvorsen, B.; Heggelund, L.; Sandberg, W.J.; Semb, A.G.; Froland, S.S.; et al. Chemokines in children with heterozygous familiar hypercholesterolemia: Selective upregulation of RANTES. Arterioscler. Thromb. Vasc. Biol. 2006, 26, 200-205. [CrossRef] [PubMed]

14. Landmesser, U.; Hornig, B.; Drexler, H. Endothelial function: A critical determinant in atherosclerosis? Circulation 2004, 109, 27-33. [CrossRef] [PubMed]

15. Olson, N.C.; Sallam, R.; Doyle, M.F.; Tracy, R.P.; Huber, S.A. T helper cell polarization in healthy people: Implications for cardiovascular disease. J. Cardiovasc. Transl. Res. 2013, 6, 772-786. [CrossRef] [PubMed]

16. Chironi, G.; Dosquet, C.; del-Pino, M.; Denarie, N.; Megnien, J.L.; Drouet, L.; Bal dit Sollier, C.; Levenson, J.; Simon, A. Relationship of circulating biomarkers of inflammation and hemostasis with preclinical atherosclerotic burden in nonsmoking hypercholesterolemic men. Am. J. Hypertens. 2006, 19, 1025-1031. [CrossRef] [PubMed]

17. Von Hundelshausen, P.; Schmitt, M.M. Platelets and their chemokines in atherosclerosis-clinical applications. Front. Physiol. 2014, 5, 294. [CrossRef]

18. Mazor, R.; Shurtz-Swirski, R.; Farah, R.; Kristal, B.; Shapiro, G.; Dorlechter, F.; Cohen-Mazor, M.; Meilin, E.; Tamara, S.; Sela, S. Primed polymorphonuclear leukocytes constitute a possible link between inflammation and oxidative stress in hyperlipidemic patients. Atherosclerosis 2008, 197, 937-943. [CrossRef]

19. Weber, C.; Shantsila, E.; Hristov, M.; Caligiuri, G.; Guzik, T.; Heine, G.H.; Hoefer, I.E.; Monaco, C.; Peter, K.; Rainger, E.; et al. Role and analysis of monocyte subsets in cardiovascular disease. Joint consensus document of the European Society of Cardiology (ESC) Working Groups "Atherosclerosis \& Vascular Biology" and “Thrombosis". Thromb Haemost 2016, 116, 626-637. [CrossRef]

20. Fadini, G.P.; Simoni, F.; Cappellari, R.; Vitturi, N.; Galasso, S.; Vigili de Kreutzenberg, S.; Previato, L.; Avogaro, A. Pro-inflammatory monocyte-macrophage polarization imbalance in human hypercholesterolemia and atherosclerosis. Atherosclerosis 2014, 237, 805-808. [CrossRef]

21. Wu, H.; Ballantyne, C.M. Dyslipidaemia: PCSK9 inhibitors and foamy monocytes in familial hypercholesterolaemia. Nat. Rev. Cardiol. 2017, 14, 385-386. [CrossRef] [PubMed]

22. Kratofil, R.M.; Kubes, P.; Deniset, J.F. Monocyte Conversion During Inflammation and Injury. Arterioscler. Thromb. Vasc. Biol. 2017, 37, 35-42. [CrossRef] [PubMed]

23. Urra, X.; Villamor, N.; Amaro, S.; Gomez-Choco, M.; Obach, V.; Oleaga, L.; Planas, A.M.; Chamorro, A. Monocyte subtypes predict clinical course and prognosis in human stroke. J. Cereb. Blood Flow Metab. 2009, 29, 994-1002. [CrossRef] [PubMed]

24. Ketelhuth, D.F.; Hansson, G.K. Adaptive Response of T and B Cells in Atherosclerosis. Circ. Res. 2016, 118, 668-678. [CrossRef] [PubMed]

25. Ding, J.W.; Zheng, X.X.; Zhou, T.; Tong, X.H.; Luo, C.Y.; Wang, X.A. HMGB1Modulates the Treg/Th17 Ratio in Atherosclerotic Patients. J. Atherosc. Thromb. 2016, 23, 737-745. [CrossRef] [PubMed]

26. Quandt, D.; Rothe, K.; Scholz, R.; Baerwald, C.W.; Wagner, U. Peripheral CD4CD8 double positive T cells with a distinct helper cytokine profile are increased in rheumatoid arthritis. PLoS ONE. 2014, 9, e93293. [CrossRef] [PubMed]

27. Yoshimoto, T. The Hunt for the Source of Primary Interleukin-4: How We Discovered That Natural Killer T Cells and Basophils Determine T Helper Type 2 Cell Differentiation In Vivo. Front. Immunol. 2018, 9, 716. [CrossRef]

28. Rius, C.; Company, C.; Piqueras, L.; Cerda-Nicolas, J.M.; Gonzalez, C.; Servera, E.; Ludwig, A.; Morcillo, E.J.; Sanz, M.J. Critical role of fractalkine (CX3CL1) in cigarette smoke-induced mononuclear cell adhesion to the arterial endothelium. Thorax 2013, 68, 177-186. [CrossRef]

29. Rius, C.; Piqueras, L.; Gonzalez-Navarro, H.; Albertos, F.; Company, C.; Lopez-Gines, C.; Ludwig, A.; Blanes, J.I.; Morcillo, E.J.; Sanz, M.J. Arterial and venous endothelia display differential functional fractalkine (CX3CL1) expression by angiotensin-II. Arterioscler. Thromb. Vasc. Biol. 2013, 33, 96-104. [CrossRef] 
30. Marques, P.; Collado, A.; Escudero, P.; Rius, C.; Gonzalez, C.; Servera, E.; Piqueras, L.; Sanz, M.J. Cigarette Smoke Increases Endothelial CXCL16-Leukocyte CXCR6 Adhesion In Vitro and In Vivo. Potential Consequences in Chronic Obstructive Pulmonary Disease. Front. Immunol. 2017, 8, 1766. [CrossRef]

31. Furio, E.; Garcia-Fuster, M.J.; Redon, J.; Marques, P.; Ortega, R.; Sanz, M.J.; Piqueras, L. CX3CR1/CX3CL1 Axis Mediates Platelet-Leukocyte Adhesion to Arterial Endothelium in Younger Patients with a History of Idiopathic Deep Vein Thrombosis. Thromb Haemost 2018, 118, 562-571. [CrossRef] [PubMed]

32. Michelson, A.D.; Barnard, M.R.; Krueger, L.A.; Valeri, C.R.; Furman, M.I. Circulating monocyte-platelet aggregates are a more sensitive marker of in vivo platelet activation than platelet surface P-selectin: Studies in baboons, human coronary intervention, and human acute myocardial infarction. Circulation 2001, 104, 1533-1537. [CrossRef] [PubMed]

(C) 2018 by the authors. Licensee MDPI, Basel, Switzerland. This article is an open access article distributed under the terms and conditions of the Creative Commons Attribution (CC BY) license (http:/ / creativecommons.org/licenses/by/4.0/). 